COMMUNICATIONS IN

ANALYSIS AND GEOMETRY

Volume 13, Number 3, 561-589, 2005

\title{
Patterson-Sullivan measures and quasi-conformal deformations
}

\author{
MARTIN BRIDGEMAN ${ }^{1}$ AND EdWARD C. TAYLOR ${ }^{2}$
}

\begin{abstract}
In this paper we relate the ergodic action of a Kleinian group on the space of line elements to the conformal action of the group on the sphere at infinity. In particular, we show that for a pair of geometrically isomorphic convex co-compact Kleinian groups, the ratio of the length of the Patterson-Sullivan measure on line element space to the length of its push-forward is bounded below by the ratio of the Hausdorff dimensions of the limit sets. Our primary techniques come from ergodic theory and Patterson-Sullivan theory.
\end{abstract}

\section{Basics and Statement of Results.}

Let Isom $_{+}\left(\mathbf{H}^{n}\right) n \geq 2$ be the space of orientation-preserving isometries of $\mathbf{H}^{n}$. As is well known, this space of isometries can be given the topology induced by uniform convergence on compact sets. A Kleinian group $\Gamma$ is a discrete subgroup of $\operatorname{Isom}_{+}\left(\mathbf{H}^{n}\right)$. As such, $\Gamma$ acts discontinuously on $\mathbf{H}^{n}$, and because we make a standing assumption that the action is torsion-free, the quotient manifold $N=\mathbf{H}^{n} / \Gamma$ is a complete Riemannian manifold of constant curvature -1 .

A Kleinian group $\Gamma$ also acts as a discrete subgroup of conformal automorphisms of the sphere at infinity $\mathbf{S}_{\infty}^{n-1}$; this action partitions $\mathbf{S}_{\infty}^{n-1}$ into two disjoint sets. The regular set $\Omega_{\Gamma}$ is the largest open set in $\mathbf{S}_{\infty}^{n-1}$ on which $\Gamma$ acts properly discontinuously, and the limit set $L_{\Gamma}$ is its complement. In the case that $L_{\Gamma}$ contains more than 2 points, the limit set is characterized as being the smallest closed $\Gamma$-invariant subset of $\mathbf{S}_{\infty}^{n-1}$. Define the convex hull $C H\left(L_{\Gamma}\right)$ of the limit set $L_{\Gamma}$ to be the smallest convex subset of $\mathbf{H}^{n}$, so that all geodesics with both limit points in $L_{\Gamma}$ are contained in $C H\left(L_{\Gamma}\right)$. We can take the quotient of $C H\left(L_{\Gamma}\right)$ by $\Gamma$ (denoted by $C(\Gamma)$ ); this is the convex core. It is the smallest convex submanifold of $N=\mathbf{H}^{n} / \Gamma$, so that

\footnotetext{
${ }^{1}$ Research supported in part by NSF grant DMS 0305634

${ }^{2}$ Research supported in part by NSF grant DMS 0305704
} 
the inclusion map is a homotopy equivalence. A Kleinian group is convex co-compact if its associated convex core is compact, and it is geometrically finite if the volume of the unit neighborhood of the convex core is finite (see Bowditch [4]). The content of this paper deals almost exclusively with convex co-compact Kleinian groups.

One can view the property of $\Gamma$ being geometrically finite as being a restriction on how the limit set is formed from an orbit $\Gamma(0), 0 \in \mathbf{H}^{n}$. The conical limit set $C L_{\Gamma}$ is the set of points $x \in L_{\Gamma}$ so that for each such point, there exists a Euclidean cone $C_{x}$ based at $x$ in $\mathbf{H}^{n}$, and an infinite sub-orbit $\left\{\gamma_{j}(0)\right\} \subset \Gamma(0)$, so that $\gamma_{j}(0) \in C_{x} \neq \emptyset$ and $\lim _{j \rightarrow \infty} \gamma_{j}(0)=x$. Given the isometric action of $\Gamma$ on $\mathbf{H}^{n}$, it is an observation that $\Gamma$ being convex co-compact implies that $L_{\Gamma}$ is purely conical, i.e. that $L_{\Gamma}=C L_{\Gamma}$. We will not consider Kleinian groups containing parabolic elements, and thus, we do not detail the dynamical restrictions on the approximation of parabolic fixed points imposed by the assumption of geometric finiteness. We also note that this paper deals exclusively with Kleinian groups having the property that vol $\mathbf{H}^{n} / \Gamma$ is unbounded (the so-called co-infinite Kleinian groups), so as to avoid situations where the Mostow Rigidity Theorem makes our results automatic. For the basics in the theory of Kleinian groups, we refer the reader to Maskit [9].

Let $\Gamma$ be a geometrically finite Kleinian group, and suppose there exists a quasi-conformal homeomorphism of $\mathbf{S}_{\infty}^{n-1}$ to itself, so that conjugation by this map results in another Kleinian group $\widehat{\Gamma}$. The Kleinian group $\widehat{\Gamma}$ is said to be quasi-conformally conjugate to $\Gamma$; note that the conjugacy induces a typepreserving isomorphism between the groups. We call such an isomorphism a geometric isomorphism. Quasi-conformal deformations extend equivariantly to quasi-isometries of $\mathbf{H}^{n}$, see [15], [12], and [8].

We now give a brief description of the parts of the Patterson-Sullivan theory needed to state the Main Theorem. For further background in the basics of Patterson-Sullivan theory, see Nicholls [10]. Let $x \in \mathbf{H}^{n}$ and give $\mathbf{S}_{\infty}^{n-1}$ the spherical metric obtained by taking the Poincaré model for $\mathbf{H}^{n}$ with $x$ as the origin. Define $\mu_{x, s}$, the $s$-dimensional Hausdorff measure on $\mathbf{S}_{\infty}^{n-1}$, as follows. If $E$ is a Borel set in $\mathbf{S}_{\infty}^{n-1}$ and $\epsilon>0$, then let

$$
\mu_{x, s}^{\epsilon}(E)=\inf \left\{\sum_{j=1}^{\infty} c_{j}^{s}: E \subset \bigcup B\left(x_{j}, c_{j}\right) ; c_{j} \leq \epsilon\right\}
$$

where $B\left(x_{j}, c_{j}\right)$ denotes an open ball centered at $x_{j} \in \mathbf{S}_{\infty}^{n-1}$ of radius $c_{j}$ in the spherical metric on $\mathbf{S}_{\infty}^{n-1}$. As $\mu_{x, s}^{\epsilon}$ is clearly non-decreasing as $\epsilon$ decreases, 
we can take the (possibly infinite) limit

$$
\mu_{x, s}(E)=\lim _{\epsilon \rightarrow 0} \mu_{x, s}^{\epsilon}(E) .
$$

Given $x, y \in \mathbf{H}^{n}$, the measures $\mu_{x}, \mu_{y}$ satisfy,

$$
\frac{d \mu_{x, s}}{d \mu_{y, s}}(\xi)=\left(\frac{P(x, \xi)}{P(y, \xi)}\right)^{s}
$$

where $P(x, \xi)=\frac{1-|x|^{2}}{|x-\xi|^{2}}$ is the Poisson kernel (see Section 1 of [13]). Therefore, it follows that

$$
e^{-s d(x, y)} \mu_{x, s}(E) \leq \mu_{y, s}(E) \leq e^{s d(x, y)} \mu_{x, s}(E)
$$

where $d(x, y)$ is the hyperbolic distance from $x$ to $y$. Therefore, we say a set $E$ has s-dimensional Hausdorf measure zero if $\mu_{x, s}(E)=0$ for any choice of $x$. Furthermore, the Hausdorff dimension $D(E)$ of a set $E \subset \mathbf{S}_{\infty}^{n-1}$ is well-defined by

$$
D(E)=\inf \left\{s: \mu_{x, s}(E)=0\right\}=\sup \left\{s: \mu_{x, s}(E)=\infty\right\} .
$$

Again fix $s \in \mathbf{R}^{+} \cup\{0\}$, and let $x, y \in \mathbf{H}^{n}$ be two fixed (chosen) points. In all that follows, we will denote the hyperbolic metric on $\mathbf{H}^{n}$ by $d$. We define the Poincaré series of a Kleinian group $\Gamma$ to be

$$
g_{s}(x, y)=\sum_{\gamma \in \Gamma} e^{-s d(x, \gamma y)}
$$

It is an easy calculation to show, for every discontinuous group acting isometrically on $\mathbf{H}^{n}$, that if $s>n-1$, then $g_{s}(x, y)<\infty$ for all $(x, y) \in \mathbf{H}^{n} \times \mathbf{H}^{n}$. Let

$$
\delta(\Gamma)=\inf \left\{s: g_{s}(x, y)<\infty\right\}
$$

then $\delta(\Gamma)$ is called the exponent of convergence of the Poincaré series. We say a group is of convergence type if its Poincaré series converges at the critical exponent and of divergence type otherwise. It is a result of Sullivan [14] and Tukia [16] that if $\Gamma$ is a geometrically finite Kleinian group having non-empty regular set, then $\delta(\Gamma)<n-1$.

Following the work of Patterson and Sullivan, a measure can be constructed on $\mathbf{S}_{\infty}^{n-1}$ which is supported on $L_{\Gamma}$ - one that is descriptive of the geometric and conformal actions of $\Gamma$. For $x, y \in \mathbf{H}^{n}$ and $s>\delta(\Gamma)$, we 
consider the measure $\sigma_{x, s}$ to have Dirac point mass of weight $\frac{e^{-s d(x, \gamma y)}}{g_{s}(y, y)}$ at each point $\gamma y$. A Patterson-Sullivan measure $\sigma_{x}$ is constructed by taking the limit as $s \rightarrow \delta^{+}(\Gamma)$ of these measures in the weak* topology. If $\Gamma$ is assumed to be geometrically finite, as will always be the case in the context of this paper, then this construction of Patterson and Sullivan yields a unique measure class (independent of the choice of $x$ and $y$.) Amongst its many properties, perhaps the most basic is that the Patterson-Sullivan measure is a finite and positive measure which is supported on the limit set (being of divergence type guarantees that the support of the Patterson-Sullivan measure is supported only on the limit set).

Patterson-Sullivan theory provides a remarkable description of the conformal action of a geometrically finite Kleinian group in terms of its Patterson-Sullivan measure. One facet of this description, restricted to the setting of interest to us, is given below.

Theorem 1.1 (Patterson [11], Sullivan [14] and Tukia [16]). Let $\Gamma$ be a non-elementary, geometrically finite Kleinian group acting on $\mathbf{H}^{n}$. Then, $\delta(\Gamma)=D\left(L_{\Gamma}\right)$ and each Patterson-Sullivan measure is supported on the limit set $L_{\Gamma}$.

Further, if $\Gamma$ is convex co-compact, then each Patterson-Sullivan measure $\sigma_{x}$ is non-atomic, and is equal to the Hausdorff measure $\mu_{x, \delta(\Gamma)}$ (up to scaling) of dimension $\delta(\Gamma)$ restricted to the limit set $L_{\Gamma}$.

Remark 1.2. One can find a complete presentation of these results, from first principles, in chapters 3 and 4 of Nicholls [10]. For instance, the most important of the results listed above, that the Patterson-Sullivan measure is a constant multiple of the Hausdorff measure, is Theorem 4.6.4 of [10].

We will relate the distortion of a convex co-compact hyperbolic structure by a quasi-isometry via the distortion of a canonical measure built from the Patterson-Sullivan measure acting on a portion of the unit tangent bundle. We now describe this canonical measure. The space of oriented geodesics $G\left(\mathbf{H}^{n}\right)$ in $\mathbf{H}^{n}$ can be identified with their endpoints in $\mathbf{S}_{\infty}^{n-1}$. Therefore, $G\left(\mathbf{H}^{n}\right) \cong\left(\mathbf{S}_{\infty}^{n-1} \times \mathbf{S}_{\infty}^{n-1}\right)$-diagonal. A measure on $G\left(\mathbf{H}^{n}\right)$ which is supported on $\left(L_{\Gamma} \times L_{\Gamma}-\right.$ diagonal $)$ and that is invariant under $\Gamma$ is called a geodesic current (see [3]). One such geodesic current is the Patterson-Sullivan geodesic current $m$, which is given differentially by

$$
d m(a, b)=\frac{d \sigma_{0}(a) d \sigma_{0}(b)}{|b-a|^{2 \delta(\Gamma)}} .
$$


Here, $\sigma_{0}$ is the Patterson-Sullivan measure based at the origin in the Poincaré model of $\mathbf{H}^{n}$, and $|\cdot|$ is the chordal distance on $\mathbf{S}_{\infty}^{n-1}$ calculated with respect to the standard metric on $\mathbf{R}^{n}$. We again consider the Poincaré model for hyperbolic space, and let $\Omega(n)=\mathbf{H}^{n} \times \mathbf{S}_{\infty}^{n-1}$. This space is the space of line elements of $\mathbf{H}^{n}$, with a pair $(x, \xi)$ representing a directed geodesic through $x$ and in the direction of $\xi$. We write $\nu_{-}$and $\nu_{+}$for the endpoints of this geodesic (with + and - specified by the orientation.) Let $z$ be the Euclidean midpoint of this geodesic, and let $s$ be the signed hyperbolic distance of $z$ to $x$. Then, we have a natural correspondence between $\Omega(n)$ and $G\left(\mathbf{H}^{n}\right) \times \mathbf{R}$ given by $(x, \xi) \rightarrow\left(\nu_{-}, \nu_{+}, s\right)$. The geodesic flow is a family of diffeomorphisms $g_{t}: \Omega(n) \rightarrow \Omega(n)$ defined by $g_{t}\left(\nu_{-}, \nu_{+}, s\right) \rightarrow\left(\nu_{-}, \nu_{+}, s+t\right)$. There is of course a natural extension of the action of $I s o m_{+}\left(\mathbf{H}^{n}\right)$ on $\mathbf{H}^{n}$ to an action on $\Omega(n)$. Let $\Gamma$ be a Kleinian group, then the geodesic flow on $\Omega(n)$ descends to the geodesic flow on the quotient line element space $\Omega(n) / \Gamma$ by virtue of the fact that it commutes with any element of $\gamma \in \Gamma$.

We will need more structure in order to define a quantity that measures the distortion of the Patterson-Sullivan geodesic current under deformation. Using the above notation, it is clear that there is a fiber map

$$
\pi: \Omega(n) \rightarrow G\left(\mathbf{H}^{n}\right)
$$

defined by $\pi\left(\nu_{-}, \nu_{+}, s\right)=\left(\nu_{-}, \nu_{+}\right)$, with the fiber over the geodesic $\left(\nu_{-}, \nu_{+}\right)$ being all line elements in $\Omega(n)$ belonging to the geodesic. Using the fiber map $\pi$ (see Lemma 4.2), we can lift any geodesic current $\mu$ to a line element measure $\mu^{*}$ on $\Omega(n)$ by taking the product of $\mu$ with the hyperbolic length measure $\lambda$, i.e.

$$
d \mu^{*}=d \mu d \lambda \text {. }
$$

Clearly, $\mu^{*}$ is also invariant under the action of $\Gamma$ on $\Omega(n)$, and so naturally descends to a measure on $\Omega(n) / \Gamma$. In particular, the Patterson-Sullivan geodesic current $m$ lifts to a measure on $\Omega(n)$, which we denote by $M=m^{*}$. (This discussion is partially reprised in Section 4.) By abuse of notation, we will also refer to the measure $M$ generated by $d m^{*}$ as the Patterson-Sullivan measure. We define the length of a geodesic current $\mu$ by $L(\mu)=\mu^{*}(\Omega(n) / \Gamma)$. We compile the crucial facts in the following theorem, as used in this paper, concerning this Patterson-Sullivan measure:

Theorem 1.3 (See Theorem 3 in [14]). Let $\Gamma$ be a convex co-compact Kleinian group. Then, geodesic flow acts ergodically on $\Omega(n)$ with respect to the measure $M$, induced from the Patterson-Sullivan geodesic current 
$m$. Furthermore, the length of the Patterson-Sullivan geodesic current $m$ is finite, i.e. $M(\Omega(n) / \Gamma)<\infty$.

Remark 1.4. A detailed discussion of both the Patterson-Sullivan geodesic current and the measure, in the context of dynamical systems, is also provided in [13], Section 4.

Let $\Gamma_{1}, \Gamma_{2}$ be quasi-conformally conjugate Kleinian groups. It is clear that a quasi-conformal deformation preserves the regular set and the limit set. Thus, we have a homeomorphism $f: L_{\Gamma_{1}} \rightarrow L_{\Gamma_{2}}$ of the limit sets induced by the quasi-conformal deformation, with inverse $g$. Let $m_{1}, m_{2}$ be the corresponding Patterson-Sullivan geodesic currents for $\Gamma_{1}, \Gamma_{2}$ respectively. We can push forward $m_{1}, m_{2}$ by the maps $f, g$ to define the distortion ratios $\mathcal{R}_{12}, \mathcal{R}_{21}$ as follows:

$$
\mathcal{R}_{12}=\frac{L_{2}\left(f_{*} m_{1}\right)}{L_{1}\left(m_{1}\right)} \quad \mathcal{R}_{21}=\frac{L_{1}\left(g_{*} m_{2}\right)}{L_{2}\left(m_{2}\right)},
$$

where $L_{1}, L_{2}$ are the length functions on the spaces of geodesic currents $\mathcal{C}\left(\Gamma_{1}\right), \mathcal{C}\left(\Gamma_{2}\right)$ respectively.

Theorem 1.5 (Main). Suppose $\Gamma_{i=1,2}$ are geometrically isomorphic, convex co-compact, co-infinite Kleinian groups. Let $\Gamma_{1}$ and $\Gamma_{2}$ have critical exponents $\delta\left(\Gamma_{1}\right)$ and $\delta\left(\Gamma_{2}\right)$ respectively. Then,

$$
\frac{1}{\mathcal{R}_{12}} \leq \frac{\delta\left(\Gamma_{2}\right)}{\delta\left(\Gamma_{1}\right)} \leq \mathcal{R}_{21}
$$

As an immediate corollary of our main result, we observe that the distortion ratio detects geometrically when a quasi-conformal deformation increases Hausdorff dimension.

Corollary 1.6. Let $\Gamma_{i=1,2}$ be as in the Main Theorem. If $\mathcal{R}_{12}<1$, then $\delta\left(\Gamma_{1}\right)<\delta\left(\Gamma_{2}\right)$.

Remark 1.7. In the case where $\Gamma_{1}, \Gamma_{2}$ are quasi-conformally conjugate Fuchsian groups, the Main Theorem implies $\mathcal{R}_{12} \geq 1$. Bonahon [3], proved this inequality using the language of Liouville geodesic currents and then used it to realize the Weil-Petersson metric on Teichmüller space. (See [17] for a comprehensive discussion of the Weil-Petersson metric on Teichmüller space.) This paper is part of a larger project to investigate whether a metric on the deformation space of a convex co-compact Kleinian group can be 
formed from the distortion ratio. Indeed, in [6], we have been able to show that there is a natural symmetric bilinear two form on quasi-Fuchsian space which extends the Weil-Petersson metric on Teichmüller space. We also note that the Main Theorem is trivially true for convex co-compact groups uniformizing finite volume hyperbolic (i.e. closed) manifolds. By the Mostow Rigidity Theorem, the only deformations of such groups are isometric deformations. Thus, the resulting ratios are identically equal to one. It is also well known that the limit set of any such group is the whole sphere $\mathbf{S}_{\infty}^{n-1}$.

Plan of Paper: In the next two sections, we continue to develop the technical definitions and results we need to both define the length distortion function and to characterize it in terms of the associated Poincaré series. Section 4 culminates in a proof of the Main Theorem (Theorem 4.14); here, we also prove the necessary geometric and analytic relationships between the associated spaces of geodesic currents defined by the two Kleinian groups. Ergodic theory, in the guise of the Birkhoff Ergodic Theorem and the ergodicity of geodesic flow, is central to the arguments in this section. We now wish to comment briefly on the connection between this paper and the contents of our previous work. In [5], we restrict ourselves to convex co-compact quasi-Fuchsian hyperbolic manifolds; in this setting, there is a natural geometric isomorphism between the Fuchsian holonomy of the hyperbolic structure on a boundary component of the convex core and the hyperbolic action of the quasi-Fuchsian group. By expanding our repertoire of techniques, we enlarge our focus from the first paper to the consideration of any two geometrically isomorphic convex co-compact Kleinian groups.

\section{Poincaré series and limit sets.}

The purpose of this section is to prove a technical result concerning the relationship between the exponent of convergence of the Poincaré series and the Hausdorff dimension of the conical limit set. Though the result and proof techniques are standard, we include a proof for completeness. Let $\left(\mathbf{H}^{n}, d\right)$ be the ball model of hyperbolic space, and let $K$ be a countable collection of Möbius transformations acting discontinuously on $\mathbf{H}^{n}$. An equivalent formulation of the usual Poincare series has a more Euclidean presentation. Fix the origin $0 \in \mathbf{H}^{n}$ and let $s \in \mathbf{R}^{+} \cup\{0\}$, define the Euclidean s-Poincaré series to be

$$
\Sigma(s, 0)=\sum_{k \in K}(1-|k(0)|)^{s}
$$


here, $|k(0)|$ is the Euclidean distance of the point $k(0)$ to the origin. (As with the familiar form of the Poincaré series, the convergence properties of $\Sigma(s, x)$ does not depend on the choice of $x \in \mathbf{H}^{n}$; see Section 1.6 of [10] for an introduction to the Euclidean $s$-Poincaré series.) Note, via the hyperbolic distance formula for a point to the origin in the ball model, we can observe that

$$
\frac{(1-|k(0)|)^{s}}{2^{s}} \leq e^{-s d(0, k(0))} \leq(1-|k(0)|)^{s}
$$

Define

$$
\delta_{\text {euc }}(K)=\inf \{s: \quad \Sigma(s, 0)<\infty\}
$$

it is thus an observation, using the inequality above, that $\delta(K)=\delta_{\text {euc }}(K)$. We thus denote the exponent of convergence by $\delta$ regardless of the choice of a hyperbolic or Euclidean presentation of the Poincaré series.

Let $C L_{K}$ be the collection of conical limit points of $K$; we assume that $K$ is such that $C L_{K} \neq \emptyset$ to avoid the trivial case. We will show:

Lemma 2.1. Let $K$ be a countable collection of Möbius transformations acting discontinously on $\mathbf{H}^{n}$. Then, $D\left(C L_{K}\right) \leq \delta(K)$; in particular, if $s>\delta(K)$, then the $s$-dimensional Hausdorff measure of $C L_{K}$ is zero.

Proof. Fix $M>0$. For $k \in K-\{\operatorname{id}\}$, denote by $B_{k}(M)$ the Euclidean ball in $\mathbf{S}_{\infty}^{n-1}$ with radius $M(1-|k(0)|)$ and center $\frac{k(0)}{|k(0)|}$. Define

$$
E_{M}=\left\{x \in \mathbf{S}_{\infty}^{n-1}: x \in B_{k}(M) \text { for infinitely many } k \in K\right\} .
$$

It is well-known that

$$
C L_{K}=\bigcup_{M=1}^{\infty} E_{M} .
$$

The proof proceeds by a covering argument that estimates, from above, the Hausdorff dimension of each $E_{M}$. Let $r>0$ be an arbitrarily chosen radial length. We have, for each fixed $M$, that

$$
\#\left\{k \in K: \operatorname{diam}\left(B_{k}(M)\right) \geq r\right\}=\#\left\{k \in K:|k(0)| \leq 1-\frac{r}{2 M}\right\}
$$

because $K$ acts discontinuously on $\mathbf{H}^{n}$, we observe that $\left\{B_{k}(M)\right.$ : $\left.\operatorname{diam}\left(B_{k}(M)\right)<r\right\}$ is a cover of $E_{M}$. Denote by $K(r)$ the subset of $K$ such that diam $\left(B_{k}(M)\right)<r$. 
Let $\delta=\delta(K)$, and fix an arbitrary $\epsilon>0$. Recalling our definition of the Hausdorff measure, let $\mu_{\delta+\epsilon}^{r}=\mu_{0, \delta+\epsilon}^{r}$. Thus,

$$
\mu_{\delta+\epsilon}^{r}\left(E_{M}\right)=\inf \left\{\sum_{j}\left(\operatorname{diam} U_{j}\right)^{\delta+\epsilon}: E_{M} \subset \bigcup_{j} U_{j}, \operatorname{diam} U_{j} \leq r\right\} .
$$

Thus,

$$
\mu_{\delta+\epsilon}^{r}\left(E_{M}\right) \leq \sum_{k \in K(r)}\left(\operatorname{diam} B_{k}(M)\right)^{\delta+\epsilon} \leq \sum_{k \in K}\left(\operatorname{diam} B_{k}(M)\right)^{\delta+\epsilon}
$$

Since $\sum_{k \in K}\left(\operatorname{diam} B_{k}(M)\right)^{\delta+\epsilon}=(2 M)^{\delta+\epsilon} \sum_{k \in K}(1-|k(0)|)^{\delta+\epsilon}<\infty$ via the definition of $E_{M}$ and the definition of $\delta$, we have that

$$
\mu_{\delta+\epsilon}^{r}\left(E_{M}\right)<\infty
$$

independent of $r$. Since $\epsilon>0$ was chosen arbitrarily, this shows that $D\left(E_{M}\right) \leq \delta(K)$ for any choice of $M$. The conclusion follows by noting that because $E_{M} \subset E_{M+1}$ and $C L(K)=\bigcup_{M=1}^{\infty} E_{M}$, we have that $D\left(C L_{K}\right) \leq \lim \sup _{M \rightarrow \infty} D\left(E_{M}\right) \leq \delta(K)$.

Remark 2.2. This is a standard argument, see [2] for instance. We remark that a much stronger fact is proven in [2], using in part the above argument: If $\Gamma$ is a non-elementary Kleinian group, then the exponent of convergence of the group is the Hausdorff dimension of the conical limit set.

\section{Length Distortion Facts.}

In all that follows, we will be keeping track of the distortion, under quasiisometry, of two convex co-compact hyperbolic structures. Suppose $\Gamma$ is a Kleinian group acting on $\mathbf{H}^{n}$; define $a(\gamma)=e^{(-d(x, \gamma x))}$, where $d$ is again the hyperbolic metric on the ball model of $\mathbf{H}^{n}$ and $x \in \mathbf{H}^{n}$ is a fixed choice of basepoint. These are the terms of the 1-Poincaré series $g_{1}(x, x)$ of $\Gamma$.

Let $i: \Gamma_{1} \rightarrow \Gamma_{2}$ be an isomorphism of Kleinian groups $\Gamma_{i}, i=1,2$, with inverse $j: \Gamma_{2} \rightarrow \Gamma_{1}$. We define $R_{12}: \Gamma_{1}-\{i d\} \rightarrow \mathbf{R}$ by

$$
R_{12}(\gamma)=\frac{d\left(x_{2}, i(\gamma) x_{2}\right)}{d\left(x_{1}, \gamma x_{1}\right)}
$$


where $x_{1}, x_{2} \in \mathbf{H}^{n}$ are fixed base points for the Poincarè series of $\Gamma_{1}, \Gamma_{2}$ respectively. Similarly, we define $R_{21}: \Gamma_{2}-\{i d\} \rightarrow \mathbf{R}$ by

$$
R_{21}(\gamma)=\frac{d\left(x_{1}, j(\gamma) x_{1}\right)}{d\left(x_{2}, \gamma x_{2}\right)}
$$

Therefore, if $a_{1}, a_{2}$ are the terms of the 1-Poincarè series of $\Gamma_{1}, \Gamma_{2}$ respectively, we have that

$$
\left[a_{1}(\gamma)\right]^{R_{12}(\gamma)}=a_{2}(i(\gamma)) \quad \text { and } \quad\left[a_{2}(\gamma)\right]^{R_{21}(\gamma)}=a_{1}(j(\gamma)) .
$$

Let $\delta\left(\Gamma_{1}\right), \delta\left(\Gamma_{2}\right)$ be the critical exponents of $\Gamma_{1}, \Gamma_{2}$, and fix $\epsilon>0$. We define the set $\Gamma_{12}^{\epsilon} \subset \Gamma_{1}$ by

$$
\Gamma_{12}^{\epsilon}=\left\{\gamma \in \Gamma_{1}: R_{12}(\gamma) \leq \frac{\delta\left(\Gamma_{1}\right)-\epsilon}{\delta\left(\Gamma_{2}\right)}\right\}
$$

Proposition 3.1. Let $\Gamma_{1}, \Gamma_{2}$ be isomorphic Kleinian groups, and fix $\epsilon>0$. Then,

$$
\sum_{\gamma \in \Gamma_{12}^{\epsilon}}\left[a_{1}(\gamma)\right]^{s}<\infty \text { for } s>\delta\left(\Gamma_{1}\right)-\epsilon
$$

Proof. If $k>\delta\left(\Gamma_{2}\right)$, then by definition

$$
\sum_{\gamma \in \Gamma_{2}}\left[a_{2}(\gamma)\right]^{k}<\infty
$$

As $a_{2}(\gamma)=\left[a_{1}(j(\gamma))\right]^{1 / R_{21}(\gamma)}$, we observe that

$$
\sum_{\gamma \in \Gamma_{2}}\left[a_{1}(j(\gamma))\right]^{k / R_{21}(\gamma)}<\infty
$$

Letting $\gamma^{\prime}=j(\gamma)$, we can change the sum to being over $\Gamma_{1}$ to get

$$
\sum_{\gamma^{\prime} \in \Gamma_{1}}\left[a_{1}\left(\gamma^{\prime}\right)\right]^{k R_{12}\left(\gamma^{\prime}\right)}<\infty
$$

Because $\Gamma_{12}^{\epsilon} \subseteq \Gamma_{1}$, we immediately see that

$$
\sum_{\gamma^{\prime} \in \Gamma_{12}^{\epsilon}}\left[a_{1}\left(\gamma^{\prime}\right)\right]^{k R_{12}\left(\gamma^{\prime}\right)} \leq \sum_{\gamma^{\prime} \in \Gamma_{1}}\left[a_{1}\left(\gamma^{\prime}\right)\right]^{k R_{12}\left(\gamma^{\prime}\right)}<\infty .
$$


Fix $\epsilon>0$. If $\gamma^{\prime} \in \Gamma_{12}^{\epsilon}$, then by definition $R_{12}\left(\gamma^{\prime}\right) \leq \frac{\delta\left(\Gamma_{1}\right)-\epsilon}{\delta\left(\Gamma_{2}\right)}$, and therefore,

$$
k R_{12}\left(\gamma^{\prime}\right) \leq \frac{k\left(\delta\left(\Gamma_{1}\right)-\epsilon\right)}{\delta\left(\Gamma_{2}\right)}
$$

for any $k>0$. Thus, if $s>\delta\left(\Gamma_{1}\right)-\epsilon$, then

$$
\sum_{\gamma \in \Gamma_{12}^{\epsilon}}\left[a_{1}(\gamma)\right]^{s}<\infty
$$

By applying Lemma 2.1, we immediately obtain:

Proposition 3.2. Let $K=\Gamma_{12}^{\epsilon}$ then $D\left(C L_{K}\right) \leq \delta\left(\Gamma_{1}\right)-\epsilon$.

Define

$$
C L_{12}=\bigcup_{n \in \mathbf{Z}^{+}} C L_{\Gamma_{12}^{\frac{1}{n}}}
$$

Via Proposition 3.1, and Lemma 2.1, we have that $C L\left(\Gamma_{12}^{\frac{1}{n}}\right)$ has $\delta\left(\Gamma_{1}\right)$ Hausdorff measure zero for all $n \in \mathbf{Z}^{+}$. Thus, we have

Corollary 3.3. The set $C L_{12}$ is of $\delta\left(\Gamma_{1}\right)$-Hausdorff measure zero.

For the remainder of this section, we assume that $\Gamma_{1}$ is both convex co-compact and is geometrically isomorphic to a (necessarily) convex cocompact Kleinian group $\Gamma_{2}$. By Theorem 1.1, the Patterson-Sullivan measure $\sigma_{x}$ associated to $\Gamma_{1}$, is equal to (up to scaling) the $\delta\left(\Gamma_{1}\right)$-dimensional Hausdorff measure $\mu_{x, \delta\left(\Gamma_{1}\right)}$. Therefore, as $C L_{12}$ is of $\delta\left(\Gamma_{1}\right)$-Hausdorff dimension zero, $C L_{12}$ is of $\sigma_{x}$ measure zero (Corollary 3.3.) As $\Gamma_{1}$ is convex co-compact, every point $\nu \in L_{\Gamma_{1}}$ is a conical limit point [1]. We define the length distortion function $R_{12}: \mathbf{H}^{n} \times \mathbf{H}^{n} \times \mathbf{S}_{\infty}^{n-1} \rightarrow \mathbf{R}$ by

$$
\tilde{R}_{12}\left(x_{1}, x_{2}, \nu\right)=\inf \left\{\liminf _{i \rightarrow \infty} R_{12}\left(\gamma_{i}\right) \mid \gamma_{i} x_{1} \rightarrow \nu \text { conically }\right\},
$$

where $x_{1}$ and $x_{2}$ are the chosen base points in the definition of $R_{12}(\gamma)$ (see equation 3.1.) We can of course define an analogous function $\tilde{R}_{21}$ for $\Gamma_{2}$. In the next lemma, we will see that the function $\tilde{R}_{12}$ is independent of the choice of base points $x_{1}, x_{2}$ and bounded below by the ratio of the exponents of convergence. 
Lemma 3.4. Let $\Gamma_{1}, \Gamma_{2}$ be convex co-compact and quasi-conformally conjugate and let $\sigma_{x}$ be the Patterson-Sullivan measure of $\Gamma_{1}$ based at $x$. Then, for any choice of $x_{1}, x_{2}, y_{1}, y_{2} \in \mathbf{H}^{n}$,

$$
\tilde{R}_{12}\left(x_{1}, x_{2}, \nu\right)=\tilde{R}_{12}\left(y_{1}, y_{2}, \nu\right)
$$

Defining $\tilde{R}_{12}: \mathbf{S}_{\infty}^{n-1} \rightarrow \mathbf{R}$, by $\tilde{R}_{12}(\nu)=\tilde{R}_{12}\left(x_{1}, x_{2}, \nu\right)$, then

$$
\tilde{R}_{12}(\nu) \geq \frac{\delta\left(\Gamma_{1}\right)}{\delta\left(\Gamma_{2}\right)} \quad \text { a.e. with respect to }\left(\sigma_{x}\right)
$$

Proof. Suppose that $\left(y_{1}, y_{2}\right)$ is another pair of base points, with $d\left(x_{1}, y_{1}\right)=$ $d_{1}$ and $d\left(x_{2}, y_{2}\right)=d_{2}$. By the triangle inequality, we observe that

$$
d\left(x_{1}, \gamma x_{1}\right)-2 d_{1} \leq d\left(y_{1}, \gamma y_{1}\right) \leq d\left(x_{1}, \gamma x_{1}\right)+2 d_{1},
$$

and

$$
d\left(x_{2}, i(\gamma) x_{2}\right)-2 d_{2} \leq d\left(y_{2}, i(\gamma) y_{2}\right) \leq d\left(x_{2}, i(\gamma) x_{2}\right)+2 d_{2} .
$$

Since $d\left(\gamma x_{1}, \gamma y_{1}\right)=d\left(x_{1}, y_{1}\right)=d_{1}$ for all $\gamma \in \Gamma_{1}$, if $\left\{\gamma_{i}\right\}$ is a sequence in $\Gamma_{1}$, then $\gamma_{i} x_{1}$ tends to $\nu \in \mathbf{S}_{\infty}^{n-1}$ conically if and only if $\gamma_{i} y_{1}$ tends to $\nu \in \mathbf{S}_{\infty}^{n-1}$ conically. Also, as $N_{1}=\mathbf{H}^{n} / \Gamma_{1}$ and $N_{2}=\mathbf{H}^{n} / \Gamma_{2}$ are quasi-isometric, then $d\left(x_{1}, \gamma_{i} x_{1}\right)$ and $d\left(x_{2}, i\left(\gamma_{i}\right) x_{2}\right)$ both tend to infinity as $\gamma_{i} x_{1}$ tends to $\nu \in \mathbf{S}_{\infty}^{n-1}$ conically. Therefore, by the above inequalities, we have that

$$
\liminf _{i \rightarrow \infty} \frac{d\left(x_{2}, i\left(\gamma_{i}\right) x_{2}\right)}{d\left(x_{1}, \gamma_{i} x_{1}\right)}=\liminf _{i \rightarrow \infty} \frac{d\left(y_{2}, i\left(\gamma_{i}\right) y_{2}\right)}{d\left(y_{1}, \gamma_{i} y_{1}\right)}
$$

Thus, taking the infimum over all such sequences gives $\tilde{R}_{12}\left(x_{1}, x_{2}, \nu\right)=$ $\tilde{R}_{12}\left(y_{1}, y_{2}, \nu\right)$. Thus, we can define $\tilde{R}_{12}(\nu)=\tilde{R}_{12}\left(x_{1}, x_{2}, \nu\right)$. Now, let $\tilde{R}_{12}(\nu)=k<\delta\left(\Gamma_{1}\right) / \delta\left(\Gamma_{2}\right)$. Then, there exists a sequence of group elements $\left\{\gamma_{i} \in \Gamma_{1}\right\}$, such that $\gamma_{i} x_{1} \stackrel{\text { conically }}{\longrightarrow} \nu$ with $k \leq \lim _{i \rightarrow \infty} R_{12}\left(\gamma_{i}\right)<\delta\left(\Gamma_{1}\right) / \delta\left(\Gamma_{2}\right)$. Therefore, $\nu \in C L_{12}$, which has $\sigma_{x}$ measure zero (see Corollary 3.3.) Therefore,

$$
\tilde{R}_{12}(\nu) \geq \frac{\delta\left(\Gamma_{1}\right)}{\delta\left(\Gamma_{2}\right)} \quad \text { a.e. with respect to }\left(\sigma_{x}\right) \text {. }
$$

Remark 3.5. We now extend $\tilde{R}_{12}$ to the line element space $\Omega(n)$. Let $p \in \Omega(n)$ and $\nu^{+} \in \mathbf{S}_{\infty}^{n-1}$ be the endpoint of the associated ray. Then, we 
define $\tilde{R}_{12}(p)=\tilde{R}_{12}\left(\nu^{+}\right)$; this function is a geodesic flow invariant function. By the definition of the measure $M_{1}$ of $\Gamma_{1}$ on $\Omega(n)$ in terms of $\sigma_{0}$, any set $A=\mathbf{H}^{n} \times O \subset \Omega(n)$ with $O$ having $\sigma_{0}$ measure zero, has $M_{1}$ measure zero. Therefore, it follows that

$$
\tilde{R}_{12}(p) \geq \frac{\delta\left(\Gamma_{1}\right)}{\delta\left(\Gamma_{2}\right)} \quad \text { a.e. with respect to }\left(M_{1}\right)
$$

\section{Geodesic Currents.}

Geodesic currents arise in the study of the collection of homotopy classes of closed geodesics. To illustrate the naturalness of geodesic currents, we start with the simplest possible construction of such an object. If $N=\mathbf{H}^{n} / \Gamma$ is a hyperbolic $n$-manifold, then consider a non-trivial and non-peripheral homotopy class $[\alpha]$ of closed curves so that the geodesic representative of $\alpha$ is a closed primitive oriented geodesic in $N$. Let $\gamma \in \Gamma$ be a representative of $\alpha$, and let fix $(\gamma)$ be its fixed point pair. Orbit fix $(\gamma)$ under the action of $\Gamma$ to produce a $\Gamma$-invariant discrete subset $A$ of $G\left(\mathbf{H}^{n}\right)=\mathbf{S}_{\infty}^{n-1} \times \mathbf{S}_{\infty}^{n-1}-$ diagonal. Place the Dirac measure on the orbit $A$. In this way, we identify this homotopy class of closed curves on $\mathbf{H}^{n} / \Gamma$ with a $\Gamma$-invariant measure on $G\left(\mathbf{H}^{n}\right)$; this is the simplest example of a geodesic current. We call a line element measure that is so associated to a closed geodesic a simple line element measure. It is clear that the length of a simple line element measure is simply the length of the associated closed geodesic.

Let $C_{c}(X)$ be the space of real-valued continuous functions with compact support in the topological space $X$. The measure $\mu$ acts on $\phi \in C_{c}(X)$ by $\mu(\phi)=\int \phi d \mu$. Recall the definition of the weak* topology on a space of measures $\mathcal{C}$ defined on $X$ : we say that $\mu_{i} \rightarrow \mu$ in the weak* topology on $\mathcal{C}$ if and only if for every $\phi \in C_{c}(X)$, then $\mu_{i}(\phi) \rightarrow \mu(\phi)$.

Definition 4.1. A geodesic current is a positive locally finite Borel measure on $G\left(\mathbf{H}^{n}\right)$ that is both invariant under the action of $\Gamma$ and supported on the set of geodesics with endpoints belonging to $L_{\Gamma}$. The set $\mathcal{C}(\Gamma)$ of geodesic currents of $\Gamma$ is endowed with the weak* topology.

We provide a detailed description of the line element space $\Omega(n)$ in the introduction. Let $M(\Omega(n))$ be the space of measures on $\Omega(n)$ endowed with the weak* topology. If $\mu$ is a geodesic current, recall that the line element measure $\mu^{*} \in M(\Omega(n)$ ) (see equation 1.4) is locally the product measure on $G\left(\mathbf{H}^{n}\right) \times \mathbf{R}$ given by $\mu \times \lambda$, where $\lambda$ is the Lebesgue measure on $\mathbf{R}$. We 
thus, have a well-defined map

$$
\pi^{*}: \mathcal{C}(\Gamma) \rightarrow M(\Omega(n))
$$

given by $\pi^{*}(\mu)=\mu^{*}$. Let $\mathcal{M}(\Gamma)$ be the image of $\mathcal{C}(\Gamma)$ under $\pi^{*}$; we endow it with the subspace topology from $M(\Omega(n))$. A flow box in $\Omega(n)=G\left(\mathbf{H}^{n}\right) \times \mathbf{R}$ is a closed set $B=D \times[a, b]$, where $D$ is a closed ball in $G\left(\mathbf{H}^{n}\right)$ and $[a, b]$ is a closed interval in $\mathbf{R}$. Therefore, the product $x \times[a, b] \subset B$ projects to a geodesic segment in $\mathbf{H}^{n}$ of length $b-a$. Furthermore, by definition of the product measure, we have $\mu^{*}(B)=\mu(D) \cdot(b-a)$. Thus, $\mathcal{M}(\Gamma)$ consists of $\Gamma$ invariant measures on $\Omega(n)$ having the following two properties: first, each line element in the support of a line element measure defines a hyperbolic geodesic with both endpoints in $L_{\Gamma}$, and secondly, each measure in $\mathcal{M}(\Gamma)$ is locally the product of the hyperbolic length measure along geodesics with a measure on the space of geodesics. We will show in the next lemma that the function $\pi^{*}$ is a homeomorphism between $C(\Gamma)$ and $\mathcal{M}(\Gamma)$. Note also that each measure $\mu^{*} \in \mathcal{M}(\Gamma)$ (necessarily $\Gamma$-invariant) descends to a measure on the quotient space $\Omega(n) / \Gamma$ which, by abuse of notation, we label by $\mu^{*}$ as well. We recall that the length of a geodesic current $\mu$ is $L(\mu)=\mu^{*}(\Omega(n) / \Gamma)$. It is an easy observation, in the case of a geodesic current induced by a closed geodesic, that the length of such a geodesic current is the length of the geodesic as one would expect.

Recall the fiber bundle map $\pi: \Omega(n) \rightarrow G\left(\mathbf{H}^{n}\right)$ defined in the introduction. The following lemma equates the space of geodesic currents with the space of line element measures.

Lemma 4.2. Let $\Gamma$ be a Kleinian group. Then, the map $\pi^{*}: \mathcal{C}(\Gamma) \rightarrow \mathcal{M}(\Gamma)$ is a homeomorphism.

Proof. We observe that $\pi^{*}$ is onto by the definition of $\mathcal{M}(\Gamma)$. We will show in the following order continuity, injectivity, and the existence of a continuous inverse.

Let $\mu_{j} \rightarrow \mu$ in $\mathcal{C}(\Gamma)$, and let $\phi: \Omega(n) \rightarrow \mathbf{R}$ be a continuous function with compact support $K \subset \Omega(n)$. We choose a partition of unity $\left\{\rho_{i}\right\}_{i=1}^{n}$ for a neighborhood of $K$ with $\operatorname{support}\left(\rho_{i}\right) \subset B_{i}$, where each $B_{i}$ is a flowbox. Then,

$$
\mu_{j}^{*}(\phi)=\int_{\Omega(n)} \phi d \mu_{j}^{*}=\sum_{i=1}^{n} \int_{B_{i}} \rho_{i} \phi d \mu_{j}^{*} .
$$

As $B_{i} \cong D_{i} \times[0,1]$, we break the integral into a double integral 


$$
\int_{\Omega(n)} \phi d \mu_{j}^{*}=\sum_{i=1}^{n} \int_{D_{i}}\left(\int_{x \times[0,1]} \rho_{i} \phi d \lambda\right) d \mu_{j} .
$$

We define $\widehat{\phi}: G\left(\mathbf{H}^{n}\right) \rightarrow \mathbf{R}$ by

$$
\widehat{\phi}_{i}(x)=\int_{x \times[0,1]} \rho_{i} \phi d \lambda .
$$

Thus,

$$
\mu_{j}^{*}(\phi)=\sum_{i=1}^{n} \mu_{j}\left(\widehat{\phi}_{i}\right) .
$$

Note that $\widehat{\phi}_{i} \in C_{c}\left(G\left(\mathbf{H}^{n}\right)\right)$. As $\mu_{j} \rightarrow \mu$, then $\mu_{j}\left(\widehat{\phi}_{i}\right) \rightarrow \mu\left(\widehat{\phi}_{i}\right)$ and by the above sum, we have $\mu_{j}^{*} \rightarrow \mu^{*}$. Thus, $\pi^{*}$ is continuous. It remains to show that $\pi^{*}$ is injective and has a continuous inverse. Let $\phi \in C_{c}\left(G\left(\mathbf{H}^{n}\right)\right)$ be a continuous function with compact support. Also let $f \in C_{c}(\mathbf{R})$ be a bump function on the real line with total integral one. Then, we define $\widehat{\phi}(g, x)=\phi(g) f(x)$. Thus, by definition of the product measure, we have that $\mu^{*}(\widehat{\phi})=\mu(\phi)\left(\int f d x\right)=\mu(\phi)$. To show that $\pi^{*}$ is injective, we let $\mu_{i}$ be two geodesic currents so that $\mu_{1} \neq \mu_{2}$. Thus, there exists a $\phi \in C_{c}\left(G\left(\mathbf{H}^{n}\right)\right)$ such that $\mu_{1}(\phi) \neq \mu_{2}(\phi)$. As $\mu_{i}^{*}(\widehat{\phi})=\mu(\phi)$, we therefore have that $\mu_{1}^{*}(\widehat{\phi}) \neq \mu_{2}^{*}(\widehat{\phi})$. Thus, $\mu^{*}$ is injective. Now, let $\mu_{i}^{*} \rightarrow \mu^{*}$, and let $\phi \in C_{c}\left(G\left(\mathbf{H}^{n}\right)\right)$. Thus, $\mu_{i}^{*}(\widehat{\phi}) \rightarrow \mu^{*}(\widehat{\phi})$. As $\mu^{*}(\widehat{\phi})=\mu(\phi)$, we have that $\mu_{i}(\phi) \rightarrow \mu(\phi)$, thus by the definition of the weak* topology, we observe that $\mu_{i} \rightarrow \mu$, and therefore, $\pi^{*}$ has a continuous inverse.

Using Lemma 4.2, we can show that the length function is a continuous map.

Proposition 4.3. Let $\Gamma$ be convex co-compact, then the length function $L: \mathcal{C}(\Gamma) \rightarrow \mathbf{R}$ is continuous.

Proof. As the map $\pi^{*}$ is continuous, we need only show that the map $\mu^{*} \rightarrow$ $\mu^{*}(\Omega(n) / \Gamma)$ is continuous. Recall, given the definition of a geodesic current and from the assumption that $\Gamma$ is convex co-compact, that the subset of $\Omega(n) / \Gamma$ on which any line element measure has support is compact. The result follows immediately from the definition of weak* convergence. 
Recall the definition (equation 1.3) of the Patterson-Sullivan geodesic current $m$, and of the induced Patterson-Sullivan line element measure $M=$ $m^{*}$. We now give a construction of a family of simple line element measures that converge to the measure $M$.

We describe the natural metric $D$ on $\Omega(n)$ given by parallel translation. Let $d$ be the hyperbolic distance function on $\mathbf{H}^{n}$ and $d_{x}$ be the spherical metric on the unit tangent space at $x \in \mathbf{H}^{n}$. Then, for $p, q \in \Omega(n)$, with basepoints $x, y \in \mathbf{H}^{n}$, we define $D(p, q)=d(x, y)+d_{y}\left(g_{y}(p), q\right)$ where $g_{y}(p)$ is the line element obtained by parallel translating $p$ to $y$. In particular, we note that $d(x, y) \leq D(p, q)$. Also, $D$ naturally descends to a metric on the quotient $\Omega(n) / \Gamma$.

Let $p \in \Omega(n) / \Gamma$, with base point $x_{p}$ and in the direction of the tangent vector $v_{p}$. We define the ray in the direction of $p$ to be the geodesic parameterized by length given by $r:[0, \infty) \rightarrow \mathbf{H}^{n} / \Gamma$ so that $r(0)=x_{p}$ and $r^{\prime}(0)=v_{p}$. We fix an embedded ball neighborhood $B$ of $x_{p}$. If, after a distance $\mathrm{t}$, the geodesic ray $r$ returns to $B$, we obtain a closed curve $r_{t}$ in $\mathbf{H}^{n} / \Gamma$ by joining the point $r(t)$ to $x_{p}=r(0)$. We let $a_{t}:\left[0, l_{t}\right] \rightarrow \mathbf{H}^{n} / \Gamma$ be the geodesic (parameterized by arclength) of length $l_{t}$ that is homotopic to $r_{t}$ with $a_{t}(0)=a_{t}\left(l_{t}\right)=x_{p}$. Then, $a_{t}$ is a closed curve through $x_{p}$ that is geodesic except at $x_{p}$.

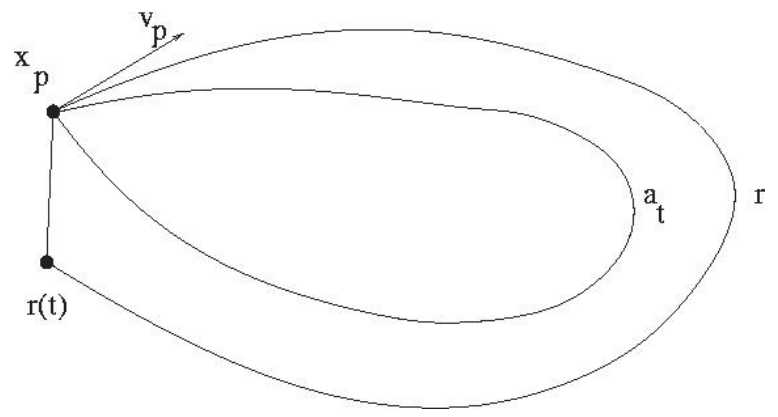

We define $R(s)=\left(r(s), r^{\prime}(s)\right)$ and $A_{t}(s)=\left(a_{t}(s), a_{t}^{\prime}(s)\right)$, and thus, obtain parameterized curves $R:[0, \infty) \rightarrow \Omega(n) / \Gamma$ and $A_{t}:[0, \infty) \rightarrow \Omega(n) / \Gamma$. As $a_{t}$ is a closed curve, the distance $D\left(A_{t}(0), A_{t}\left(l_{t}\right)\right)$ is just the angle of $a_{t}$ at $x_{p}$. The lemma below says that if the ray $r$ returns to a small neighborhood of the initial line element in the $D$ metric on $\Omega(n) / \Gamma$, then $D\left(A_{t}(0), A_{t}\left(l_{t}\right)\right)$ is small. The proof is an elementary exercise in hyperbolic geometry that we omit for the sake of brevity.

Lemma 4.4. Given $\epsilon>0$, there exist constants $\delta>0$ and $K>0$, 
each depending on $\epsilon$, such that if $t>K$ and $D(R(0), R(t))<\delta$, then $D\left(A_{t}(0), A_{t}\left(l_{t}\right)\right)<\epsilon$.

The next lemma shows that for sufficiently large $t$, if the angle of $a_{t}$ is small, then $a_{t}$ is homotopic to a closed geodesic by a bounded homotopy. Recall the definition of a bounded homotopy: let $X$ be a Riemannian manifold, then a homotopy $H:[0,1] \times[0,1] \rightarrow X$ has bounded height if there exists a $K$ such that for each $s \in[0,1]$ the curve $p_{s}(t)=H(s, t)$ have length less than or equal to $K$. The minimum value of $K$ is the height of the homotopy, a homotopy for which a height can be found is called a bounded homotopy.

Lemma 4.5. Assume that $\Gamma$ is a purely loxodromic Kleinian group. Given any $\epsilon<\pi$, the following is true: There exists a constant $K$ so that if $\alpha:[0, t] \rightarrow \mathbf{H}^{n} / \Gamma$ is a geodesic parameterized by arc length with $t>K$, and if

$$
\begin{aligned}
& \text { 1. } \alpha(0)=\alpha(t)=x, \\
& \text { 2. } d_{x}\left(\alpha^{\prime}(0), \alpha^{\prime}(t)\right)<\epsilon,
\end{aligned}
$$

then $\alpha$ is homotopic to a closed geodesic by a bounded homotopy of height less than or equal to $\frac{K}{2}$.

Proof. We will show that $K=2 \cosh ^{-1}\left(\frac{\pi}{\pi-\epsilon}\right)$ works.

Let $\pi: \mathbf{H}^{n} \rightarrow \mathbf{H}^{n} / \Gamma$ be the covering map, and let $x_{0} \in \mathbf{H}^{n}$ belong to $\pi^{-1}(x)$. Let $\tilde{\alpha}:[0, t] \rightarrow \mathbf{H}^{n}$ be the unique lift of $\alpha$ starting at $x_{0}$. Denote by $x_{t} \in \pi^{-1}(x)$ the point $x_{t}=\tilde{\alpha}(t)$. Let $\theta$ be the angle between the tangent vectors $\alpha^{\prime}(0)$ and $\alpha^{\prime}(t)$ at $x$. We let $\gamma \in \Gamma$ be the (loxodromic) element such that $x_{t}=\gamma\left(x_{0}\right)$, and let $\tilde{\beta}$ be the axis of $\gamma$. We denote by $\langle\gamma\rangle$ the cyclic group generated by $\gamma$. We orthogonally project $\left(x_{0}, x_{t}\right)$ to the points $\left(y_{0}, y_{t}\right) \in \tilde{\beta}$, and denote by $D$ the distance $d\left(x_{0}, y_{0}\right)=d\left(x_{t}, y_{t}\right)$. Thus, $\gamma\left(y_{0}\right)=y_{t}$ and therefore, the geodesic segment $\left[y_{0}, y_{t}\right] \subset \tilde{\beta}$ projects to a closed geodesic $\beta$ in $\mathbf{H}^{n} /\langle\gamma\rangle$. The convexity of the length function in hyperbolic space implies that the geodesic arc segment $\left[x_{0}, x_{t}\right] \subset \mathcal{N}_{D}\left(\left[y_{0}, y_{t}\right]\right)$ (here, the symbol $\mathcal{N}_{D}$ refers to the radius $D$ neighborhood). Therefore, by taking orthogonal projection, we obtain a homotopy of the geodesic arc $\left[x_{0}, x_{t}\right]$ to $\left[y_{0}, y_{t}\right]$ by a homotopy of height at most $D$. Descending to the quotient space $\mathbf{H}^{n} / \Gamma$, we obtain a homotopy of $\alpha$ to $\beta$ of height at most $D$.

Our goal is to show that $D$ is less than or equal to $\frac{K}{2}$, and to do so, we use the simplicial surface techniques of Thurston. We now describe 
a simplicial annulus $A$ with boundary components $\alpha$ and $\beta$. We form a simplicial quadrilateral $Q$ in $\mathbf{H}^{n}$ with vertices $x_{0}, x_{t}, y_{0}, y_{t}$ which is the union of two triangles $T_{1}, T_{2}$. The triangle $T_{1}$ has sides $x_{0} y_{0}, x_{0} x_{t}, x_{t} y_{0}$ and $T_{2}$ has sides $y_{0} y_{t}, y_{t} x_{t}, x_{t} y_{0}$. Let $Q=T_{1} \cup T_{2}$. Then, $Q$ is a quadrilateral and $Q$ projects to an annulus $A$ in $\mathbf{H}^{n} /\langle\gamma\rangle$ with boundary components $\alpha$ and $\beta$. At the vertex $x \in A$, the exterior angle is $\theta$. Therefore, the interior angle $\theta_{x}$ in $A$ at $x$ satisfies $\theta_{x} \geq \pi-\theta>\pi-\epsilon$.

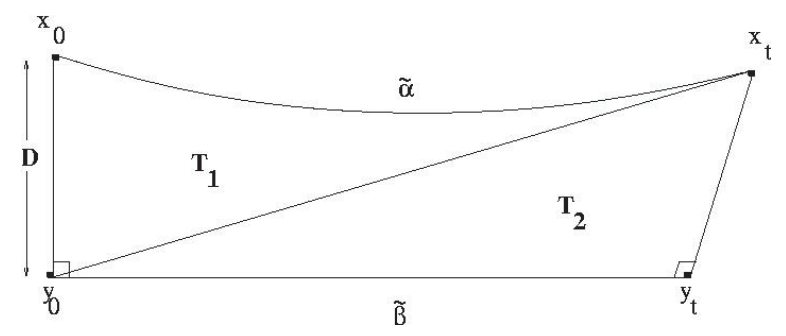

If $D>t / 2$, we let $S$ be the set of points within a distance $t / 2$ of $x$ in $A$. As $D>t / 2$, then $S$ is an embedded hyperbolic sector of angle $\theta_{x}$ and radius $t / 2$. Thus,

$$
\operatorname{Area}(A) \geq \operatorname{Area}(S) \geq \frac{\theta_{x}}{2 \pi}\left(4 \pi \sinh ^{2}(t / 4)\right)=2 \theta_{x} \sinh ^{2}(t / 4) .
$$

As the interior angles of $Q$ at $y_{0}$ and $y_{t}$ are at least $\pi / 2$, we have $\operatorname{Area}(A) \leq$ $\pi-\theta_{x}$. Therefore, $2 \theta_{x} \sinh ^{2}(t / 4) \leq \pi-\theta_{x}$ giving $\theta_{x}\left(1+2 \sinh ^{2}(t / 4)\right) \leq \pi$. As $\cosh (2 x)=1+2 \sinh ^{2}(x)$, we get

$$
\cosh (t / 2) \leq \frac{\pi}{\theta_{x}} \leq \frac{\pi}{\pi-\epsilon}
$$

Thus, $t \leq K$, which gives us a contradiction. Therefore, $D \leq t / 2$. We now let $S$ be the set of points within a distance $D$ of $x$ in $A$. Then, $S$ is an embedded hyperbolic sector of angle $\theta_{x}$ and radius $D$. Thus, as above, we have

$$
D \leq \cosh ^{-1}\left(\frac{\pi}{\pi-\epsilon}\right)=\frac{K}{2}
$$

and therefore, $\alpha$ is homotopic to $\beta$ via a homotopy of height at most $K / 2$.

Remark 4.6. In fact, the above proof shows more: the height of the homotopy tends to zero as the angle of deflection tends to zero.

A direct corollary of the above facts is: 
Corollary 4.7. There exist constants $\delta, K_{1}$, and $K_{2}$, all greater than zero, so that if $t>K_{1}$ and $D(R(0), R(t)) \leq \delta$, then $a_{t}$ is homotopic to a closed geodesic $\alpha_{(t, p)}$ via a homotopy with height bounded by $K_{2}$.

Proof. Fix $\epsilon \in(0, \pi)$. By Lemma 4.4, there are constants $\delta$ and $k_{1}$ such that if $D(R(0), R(t)) \leq \delta$ and $t>k_{1}$, then $a_{t}$ has angle at most $\epsilon$ at $x_{p}$. Let $l_{t}$ be the length of $a_{t}$; then, applying Lemma 4.5 insures that there exists a constant $k_{2}$ (depending only on $\epsilon$ ) such that for $l_{t}>k_{2}$, the geodesic curve $a_{t}$ is boundedly homotopic to its geodesic representative $\alpha_{(t, p)}$. We let $K_{2}=k_{2} / 2$. To finish the proof, we need to choose a constant $K_{1}$ such that if $t>K_{1}$, then both $t>k_{1}$ and $l_{t}>k_{2}$. By the triangle inequality, we have that $\left|l_{t}-t\right| \leq \delta$, thus the quantity $K_{1}=\max \left\{k_{1}, k_{2}+\delta\right\}$ works.

We now use the geodesics $\alpha_{\left(t_{i}, p\right)}$ to approximate the Patterson-Sullivan geodesic current $m$.

Theorem 4.8. Let $\Gamma$ be convex co-compact, and denote by $M$ the Patterson-Sullivan measure on $\Omega(n) / \Gamma$. Then, for almost every $p \in \Omega(n)$ with respect to $M$, there exists a sequence $t_{i}$ such that

$$
\lim _{t_{i} \rightarrow \infty} \frac{\alpha_{\left(t_{i}, p\right)}^{*}}{L\left(\alpha_{\left(t_{i}, p\right)}^{*}\right)}=\frac{M}{M(\Omega(n) / \Gamma)}
$$

Proof. Recall the statement of Theorem 1.3, in particular, that the Patterson-Sullivan line element measure $M$ assigns finite mass to $\Omega(n) / \Gamma$ and that the geodesic flow on $\Omega(n) / \Gamma$ is ergodic with respect to the measure $M$ (see [14].) Thus, by the Birkhoff Ergodic Theorem, given $\phi \in C_{c}(\Omega(n) / \Gamma)$, there exists a set $A_{\phi} \subset \Omega(n) / \Gamma$ of full $M$-measure such that for all $p \in A_{\phi}$

$$
\lim _{t \rightarrow \infty} \frac{1}{t} \int_{0}^{t} \phi\left(g_{s}(p)\right) d s=\frac{M(\phi)}{M(\Omega(n) / \Gamma)} .
$$

To prove that there is a set $A$ of full $M$-measure in $\Omega(n) / \Gamma$ for which the above holds for every continuous function with compact support, we choose a countable dense subset $\left\{\phi_{i}\right\}$ of the space of continuous functions with compact support on $\Omega(n) / \Gamma$ (with the $\|\cdot\|_{\infty}$ normed topology). Therefore, as the countable union of measure zero sets is of measure zero, we have that 
there is a set $A \subset \Omega(n) / \Gamma$ of full $M$-measure, such that for all $p \in A$

$$
\lim _{t \rightarrow \infty} \frac{1}{t} \int_{0}^{t} \phi_{i}\left(g_{s}(p)\right) d s=\frac{M\left(\phi_{i}\right)}{M(\Omega(n) / \Gamma)} .
$$

Let $\phi$ be any continuous function with compact support in $\Omega(n) / \Gamma$, then there exists a subsequence $\left\{\phi_{j}\right\} \subset\left\{\phi_{i}\right\}$ such that $\phi_{j} \rightarrow \phi$ in the $\|\cdot\|_{\infty}$ norm. An elementary bit of analysis shows that

$$
\lim _{t \rightarrow \infty} \frac{1}{t} \int_{0}^{t} \phi\left(g_{s}(p)\right) d s=\frac{M\left(\phi_{i}\right)}{M(\Omega(n) / \Gamma)}
$$

for all $p \in A$. Note that as $M$ is supported on those line elements associated to the convex core of $\mathbf{H}^{n} / \Gamma$, we can assume that the points of $A$ are line elements of this type. Define the ray $R:[0, \infty) \rightarrow \Omega(n)$ by $R(t)=g_{t}(p)$ for $p \in A$.

As the geodesic flow is ergodic with respect to $M$, the geodesic flow is recurrent for almost every $p \in A$. We fix a flow box about $R(0)$ so that the diameter of the flow box is smaller than the minimum value of the injectivity radius function on $\mathbf{H}^{n} / \Gamma$. Using the recurrence property of the geodesic flow, we can form a sequence of homotopically non-trivial curves by "closing up" the ray $R(s)$ each time it returns to the flow box (recurrence insures that there is an infinite sequence of distances $s$ for which return of the ray to the flow box is achieved.) From Corollary 4.7, there exist constants $\delta, K_{1}$ and $K_{2}$ such that if $t>K_{1}$ and $D(R(0), R(t)) \leq \delta$, then $a_{t}$ is homotopic via a bounded homotopy of height $K_{2}$ to the closed geodesic $\alpha_{(t, p)}$ in its homotopy class, (we can without loss of generality assume that $\delta$ is less than the diameter of the flow box.) By the recurrence property, there exists a sequence of distances $\left\{t_{i}\right\}$ from $R(0)$, with $t_{i} \rightarrow \infty$, such that $t_{i}>K_{1}$ and $D\left(R(0), R\left(t_{i}\right)\right) \leq \delta$ for all index $i$. We therefore obtain a sequence $\left\{a_{i}=a_{t_{i}}\right\}$ of geodesics, along with the associated sequence $\left\{\alpha_{i}=\alpha_{\left(t_{i}, p\right)}\right\}$ of closed geodesics (here, of course, $a_{t_{i}}$ is homotopic to $\left.\alpha_{\left(t_{i}, p\right)}\right)$. We let $L_{i}$ be the length of $\alpha_{i}$, and we let $A_{i}:\left[0, L_{i}\right] \rightarrow \Omega(n) / \Gamma$ be the closed curve given by taking line elements along the geodesic $\alpha_{i}$. We consider the covering map $p: \Omega(n) \rightarrow \Omega(n) / \Gamma$, and let $\tilde{\phi}$ be the pullback of $\phi \in C_{c}(\Omega(n) / \Gamma)$ given by $\tilde{\phi}=\phi \circ p$. Similarly, choose $\tilde{R}:[0, \infty) \rightarrow \Omega(n)$ to be a lift of $R$ and let $\tilde{A}_{i}:\left[0, L_{i}\right] \rightarrow \Omega(n)$ be the lift of $A_{i}$ corresponding to the choice of $\tilde{R}$ via the homotopy between $a_{i}$ and $\alpha_{i}$. Lifting the integral with respect to the covering map $p$, we get both

$$
\frac{1}{t} \int_{0}^{t} \phi(R(s)) d s=\frac{1}{t} \int_{0}^{t} \tilde{\phi}(\tilde{R}(s)) d s
$$


and

$$
\frac{\alpha_{i}^{*}}{L_{i}}(\phi)=\frac{1}{L_{i}} \int_{0}^{L_{i}} \tilde{\phi}\left(\tilde{A}_{i}(s)\right) d s
$$

(recall that $\alpha_{i}^{*}$ is the line element measure induced by the simple geodesic current defined by $\alpha_{i}$.) We will now show that

$$
\lim _{i \rightarrow \infty} \frac{1}{t_{i}} \int_{0}^{t_{i}} \tilde{\phi}(\tilde{R}(s)) d s=\lim _{i \rightarrow \infty} \frac{1}{L_{i}} \int_{0}^{L_{i}} \tilde{\phi}\left(\tilde{A}_{i}(s)\right) d s .
$$

We start with the elementary observation that if $f$ is a bounded function, $K$ a non-negative constant, and for each $t \in \mathbf{R}$, let $c_{t}, d_{t}$ be a pair of of real numbers so that $\left|c_{t}\right|$ and $\left|d_{t}\right|$ are both less than or equal to $K$ for all $t \in \mathbf{R}$, then

$$
\lim _{t \rightarrow \infty} \frac{1}{t} \int_{0}^{t} f(s) d s=\lim _{t \rightarrow \infty} \frac{1}{t} \int_{c_{t}}^{t+d_{t}} f(s) d s
$$

Under the fiber bundle map $\pi: \Omega(n) \rightarrow \mathbf{H}^{n}$, the curve $\tilde{A}_{i}$ is mapped to a geodesic arc $\tilde{\alpha}_{i}:\left[0, L_{i}\right] \rightarrow \mathbf{H}^{n}$ and the ray $\tilde{R}$ is mapped to a geodesic ray that we label by $\tilde{r}:[0, \infty) \rightarrow \mathbf{H}^{n}$. We orthogonally project $\tilde{r}$ onto the axis $\tilde{\alpha}_{i}$. By the description of the homotopy from $a_{i}$ to $\alpha_{i}$, it follows that the point $x=$ $\tilde{r}(0)$ projects to the point $x_{i}^{\prime}=\tilde{\alpha}_{i}(0)$. We let $y_{i}=\tilde{r}\left(t_{i}\right)$, and let the projection of this point to $\tilde{\alpha}_{i}$ be denoted by $y_{i}^{\prime}$. For each $s$, let $f(s)$ be the distance from $x_{i}^{\prime}$ on $\tilde{\alpha}_{i}$ to the projection of the point $\tilde{r}(s)$. Therefore, the point $\tilde{r}(s)$ is projected to the point $\tilde{\alpha}_{i}(f(s))$, and as orthogonal projection is distance decreasing, then $f$ is a continuous distance decreasing homeomorphism onto its image. As $y_{i}$ is within a distance $\delta$ of a point that projects to $\tilde{\alpha}_{i}\left(L_{i}\right)$, we have that $\left|f\left(t_{i}\right)-L_{i}\right| \leq \delta$. Using the fact that $a_{i}$ is homotopic to $\alpha_{i}$ via a homotopy whose height is bounded by $K_{2}$ then by the triangle inequality, we have that $d\left(x, x_{i}^{\prime}\right) \leq C$ and $d\left(y_{i}, y_{i}^{\prime}\right) \leq C$, where $C=K_{2}+\delta$. In particular, we have that $\left|L-t_{i}\right| \leq 2 C$, and thus, we have asymptotically two relatively long geodesic arcs having endpoints within a bounded distance from each other.

It follows from elementary hyperbolic geometry (see Theorem 2.4.6 in [7]) that, given any $\delta_{1}>0$, there exists a $k_{1}>0$ so that $d(\tilde{r}(s), \tilde{\alpha}(f(s))) \leq \delta_{1}$ for $k_{1} \leq s \leq s-k_{1}$. It follows similarly that the tangent vectors are close, and we get that there exists a constant $k_{2}>0$ so that $D\left(\tilde{R}(s), \tilde{A}_{i}(f(s))\right) \leq \delta_{1}$ for $k_{2} \leq s \leq t-k_{2}$.

The function $f$ is obviously differentiable, and it has the following properties. As $f$ is distance decreasing, then $f(s) \leq s$ and $f^{\prime}(s) \leq 1$. Letting $D(s)=D(\tilde{R}(s), \tilde{A}(f(s)))$, then we observe that as $D(s)$ tends to zero the 
function $f^{\prime}(s)$ tends uniformly to one. Therefore, there is a monotonically increasing function $g$ satisfying $g(x) \rightarrow 1$ as $x \rightarrow 0$ such that

$$
\frac{1}{g(D(s))} \leq f^{\prime}(s) \leq 1 \text {. }
$$

Fix $\epsilon>0$. As $\phi$ has compact support in $\Omega(n) / \Gamma$, it is uniformly continuous. Therefore, $\tilde{\phi}$ is uniformly continuous, and thus, there exists a $\delta_{1}>0$ so that $|\tilde{\phi}(p)-\tilde{\phi}(q)| \leq \epsilon$ whenever $D(p, q) \leq \delta_{1}$, where $p$ and $q$ are in the support of $\tilde{\phi}$. We can, without loss of generality, further assume that $\delta_{1} \leq \epsilon$. Compiling the information above, we thus have that

$$
\left|\tilde{\phi}(\tilde{R}(s))-\tilde{\phi}\left(\tilde{A}_{i}(f(s))\right)\right| \leq \epsilon \text { for } k_{2} \leq s \leq t_{i}-k_{2}
$$

and thus,

$$
\left|\int_{k_{2}}^{t_{i}-k_{2}} \tilde{\phi}(\tilde{R}(s)) d s-\int_{k_{2}}^{t_{i}-k_{2}} \tilde{\phi}\left(\tilde{A}_{i}(f(s))\right) d s\right| \leq \epsilon t_{i} .
$$

Letting $u=f(s)$, and as $D\left(\tilde{R}(s), \tilde{A}_{i}(f(s))\right) \leq \delta_{1}$ and using the monotonicity of $g$, we obtain for $k_{2} \leq s \leq t-k_{2}$ that

$$
\frac{1}{g\left(\delta_{1}\right)} \leq \frac{d u}{d s} \leq 1
$$

Thus:

$$
\begin{aligned}
\int_{f\left(k_{2}\right)}^{f\left(t_{i}-k_{2}\right)} \tilde{\phi}\left(\tilde{A}_{i}(u)\right) d u & \leq \int_{k_{2}}^{t_{i}-k_{2}} \tilde{\phi}\left(\tilde{A}_{i}(f(s))\right) d s \\
& \leq g\left(\delta_{1}\right) \int_{f\left(k_{2}\right)}^{f\left(t_{i}-k_{2}\right)} \tilde{\phi}\left(\tilde{A}_{i}(u)\right) d u .
\end{aligned}
$$

Combining inequalities 4.2 and 4.3 , we have that

$$
\begin{aligned}
& \left|\int_{k_{2}}^{t_{i}-k_{2}} \tilde{\phi}(\tilde{R}(s)) d s-\int_{f\left(k_{2}\right)}^{f\left(t_{i}-k_{2}\right)} \tilde{\phi}\left(\tilde{A}_{i}(s)\right) d s\right| \\
& \leq \epsilon t_{i}+\left(g\left(\delta_{1}\right)-1\right)\left|\int_{f\left(k_{2}\right)}^{f\left(t_{i}-k_{2}\right)} \tilde{\phi}\left(\tilde{A}_{i}(s)\right) d s\right| .
\end{aligned}
$$

As $\phi \in C_{c}(\Omega(n) / \Gamma)$ has compact support, there exists a constant $k_{3}$ so that $|\tilde{\phi}(p)| \leq k_{3}$ for all $p \in \Omega(n)$. Also, as $f$ is distance non-increasing, i.e. $0 \leq f(x) \leq x$, then

$$
\left|\int_{k_{2}}^{t_{i}-k_{2}} \tilde{\phi}(\tilde{R}(s)) d s-\int_{f\left(k_{2}\right)}^{f\left(t_{i}-k_{2}\right)} \tilde{\phi}\left(\tilde{A}_{i}(s)\right) d s\right| \leq \epsilon t_{i}+\left(g\left(\delta_{1}\right)-1\right) t_{i} k_{3} .
$$


Observe that $0 \leq f\left(k_{2}\right) \leq k_{2}$; also, by the triangle inequality, $\mid f\left(t_{i}-k_{2}\right)-$ $L_{i}|\leq| f\left(t_{i}-k_{2}\right)-f\left(t_{i}\right)|+| f\left(t_{i}\right)-L_{i} \mid \leq k_{2}+\delta$. Thus, there are constants $c_{i}$ and $d_{i}$ such that $\max \left\{\left|c_{i}\right|,\left|d_{i}\right|\right\} \leq k_{2}+\delta$, and so

$$
\left|\int_{k_{2}}^{t_{i}-k_{2}} \tilde{\phi}(\tilde{R}(s)) d s-\int_{c_{i}}^{L_{i}-d_{i}} \tilde{\phi}\left(\tilde{A}_{i}(s)\right) d s\right| \leq \epsilon t_{i}+\left(g\left(\delta_{1}\right)-1\right) t_{i} k_{3} .
$$

As $\left|L_{i}-t_{i}\right| \leq 2 C$, it follows that $\lim _{i \rightarrow \infty} \frac{L_{i}}{t_{i}}=1$, and therefore,

$$
\lim _{i \rightarrow \infty}\left|\frac{1}{t_{i}} \int_{0}^{t_{i}} \tilde{\phi}(\tilde{R}(s)) d s-\frac{1}{L_{i}} \int_{0}^{L_{i}} \tilde{\phi}\left(\tilde{A}_{i}(s)\right) d s\right| \leq \epsilon+\left(g\left(\delta_{1}\right)-1\right) k_{3} .
$$

As $\epsilon$ is arbitrary, we can let $\epsilon \rightarrow 0$, and thus, as $\delta_{1} \leq \epsilon$, we have $\delta_{1} \rightarrow 0$. As $\delta_{1} \rightarrow 0$, we have $g\left(\delta_{1}\right) \rightarrow 1$, and thus:

$$
\lim _{i \rightarrow \infty} \frac{1}{t_{i}} \int_{0}^{t_{i}} \tilde{\phi}(\tilde{R}(s)) d s=\lim _{i \rightarrow \infty} \frac{1}{L_{i}} \int_{0}^{L_{i}} \tilde{\phi}\left(\tilde{A}_{i}(s)\right) d s
$$

and therefore,

$$
\lim _{i \rightarrow \infty} \frac{1}{L_{i}} \int_{0}^{L_{i}} \phi\left(A_{i}(s)\right) d s=\frac{M(\phi)}{M(\Omega(n) / \Gamma)}
$$

Thus,

$$
\lim _{i \rightarrow \infty} \frac{\alpha_{i}^{*}}{L_{i}}=\frac{M}{M(\Omega(n) / \Gamma)} .
$$

Therefore, for almost every $p \in \Omega(n)$ with respect to $M$, there exist a sequence $t_{i}$ such that

$$
\lim _{i \rightarrow \infty} \frac{\alpha_{\left(t_{i}, p\right)}^{*}}{L_{\left(t_{i}, p\right)}}=\frac{M}{M(\Omega(n) / \Gamma)}
$$

We can immediately reformulate the theorem above in terms of geodesic currents using Lemma 4.2.

Corollary 4.9. Let $\Gamma$ be a convex co-compact group. Then, for almost every $p \in \Omega(n)$ with respect to $M$, there exists a sequence $t_{i} \rightarrow \infty$ such that the resulting sequence $\left\{\alpha_{\left(t_{i}, p\right)}\right\}$ of simple geodesic currents satisfies

$$
\lim _{i \rightarrow \infty} \frac{\alpha_{\left(t_{i}, p\right)}}{L\left(\alpha_{\left(t_{i}, p\right)}\right)}=\frac{m}{M(\Omega(n) / \Gamma)}
$$


Recall that $\Gamma_{1}$ and $\Gamma_{2}$ are two quasi-conformally conjugate convex cocompact Kleinian groups. Define the push-forward $f_{*}: \mathcal{C}\left(\Gamma_{1}\right) \rightarrow \mathcal{C}\left(\Gamma_{2}\right)$ of $\mu \in \mathcal{C}\left(\Gamma_{1}\right)$ by the induced homeomorphism $f: L_{\Gamma_{1}} \rightarrow L_{\Gamma_{2}}$ by $\left(f_{*} \mu\right)(\phi)=$ $\mu(\phi \circ f) ; \phi \in C_{c}\left(G\left(\mathbf{H}^{n}\right)\right)$. The map $f$ extends to a quasi-isometric (i.e. biLipschitz) map $\bar{f}: \mathbf{H}^{n} \rightarrow \mathbf{H}^{n}$ conjugating $\Gamma_{1}$ to $\Gamma_{2}$ (see [8] and [12]). Observe that if $\alpha$ is a geodesic current corresponding to a closed geodesic $\gamma$, then $f_{*} \alpha$ is the geodesic current corresponding to the unique closed geodesic in the homotopy class of the closed curve $\bar{f}(\gamma)$.

Lemma 4.10. The map $f_{*}: \mathcal{C}\left(\Gamma_{1}\right) \rightarrow \mathcal{C}\left(\Gamma_{2}\right)$ is a homeomorphism.

Proof. We first observe that because $f_{*}$ is functorial, we have that $f_{*}$ is one to one and onto. Let $\mu_{i} \rightarrow \mu$ in $\mathcal{C}\left(\Gamma_{1}\right)$ and $\phi: G\left(\mathbf{H}^{n}\right) \rightarrow \mathbf{R}$ be a continuous function with compact support $K$. Then, $f_{*} \mu(\phi)=\mu(\phi \circ f)$. The support of $\phi \circ f$ is $f^{-1}(K)$ which is compact as $f$ is a homeomorphism. As $\mu_{i} \rightarrow \mu$ and $\phi \circ f$ is continuous with compact support, we have that $\mu_{i}(\phi \circ f) \rightarrow \mu(\phi \circ f)$ and therefore, $f_{*} \mu_{i} \rightarrow f_{*} \mu$. Therefore, $f_{*}$ is continuous and similarly $g_{*}$ is continuous, where $g=f^{-1}$. Composing $f_{*}, g_{*}$, we see that $f_{*}$ has $g_{*}$ as its inverse and is therefore, a homeomorphism.

We define the distortion of a current $\mu \in \mathcal{C}\left(\Gamma_{1}\right)$ by

$$
\bar{R}_{12}(\mu)=\frac{L_{2}\left(f_{*} \mu\right)}{L_{1}(\mu)}
$$

where $L_{1}, L_{2}$ are the length functions on $\mathcal{C}\left(\Gamma_{1}\right), \mathcal{C}\left(\Gamma_{2}\right)$ respectively and $f$ is a quasi-conformal deformation inducing an isomorphism taking a convex co-compact Kleinian group $\Gamma_{1}$ to $\Gamma_{2}$. The distortion $\bar{R}_{21}(\mu)$ of a current $\mu \in \mathcal{C}\left(\Gamma_{2}\right)$ is defined analogously.

In terms of the induced line element measures, the distortion is given by

$$
\bar{R}_{12}(\mu)=\frac{\left(f_{*} \mu\right)^{*}\left(\Omega(n) / \Gamma_{2}\right)}{\mu^{*}\left(\Omega(n) / \Gamma_{1}\right)}
$$

Because geodesic flow acting on $\Omega(n)$ is ergodic with respect to $M_{1}$ for convex co-compact Kleinian groups, and since $\tilde{R}_{12}(p)$ is a measurable, geodesic flow invariant function (Remark 3.5), we know that $\tilde{R}_{12}$ is constant on $\Omega(n)$ almost everywhere $\left(M_{1}\right)$. The following lemma uses the compactness of the convex core to relate this constant to the distortion of the Patterson-Sullivan geodesic current $m_{1}$. 
Lemma 4.11. Let $\Gamma_{i}, i=1,2$ be convex co-compact, quasi-conformally conjugate Kleinian groups. Then,

$$
\tilde{R}_{12}(p) \leq \bar{R}_{12}\left(m_{1}\right) \quad \text { a.e. }\left(M_{1}\right) .
$$

Proof. By Corollary 4.9, there is a set of full measure $A \subset \Omega(n)$ with respect to $M_{1}$, such that for each $p \in A$, there exists a sequence $t_{i}$ such that the Patterson-Sullivan geodesic current can be approximated using $\alpha_{\left(t_{i}, p\right)}$. By definition, we have, if $p \in \Omega(n)$ with endpoint $\nu^{+}$, that

$$
\tilde{R}_{12}(p)=\inf \liminf _{\gamma x_{1} \stackrel{\text { conical }}{\longrightarrow} \nu^{+}} R_{12}(\gamma)=\inf \liminf _{\gamma x_{1} \stackrel{\text { conical }}{\longrightarrow} \nu^{+}} \frac{d\left(x_{2}, i(\gamma) x_{2}\right)}{d\left(x_{1}, \gamma x_{1}\right)}
$$

where $x_{1}, x_{2}$ are any choice of basepoints for the 1-Poincaré series of $\Gamma_{1}$ and $\Gamma_{2}$ respectively. For any $\gamma \in \Gamma_{1}-\{\mathrm{id}\}$, we join the point $x_{1}$ to the point $\gamma\left(x_{1}\right)$. In the manifold $\mathbf{H}^{n} / \Gamma_{1}$, this curve projects to a homotopically non-trivial closed curve $a_{\gamma}$; we let $\alpha_{\gamma}$ be the unique closed geodesic ( $\Gamma_{1}$ is convex cocompact) in its homotopy class. Let $p$ have basepoint $x$, and choose $x_{1}=x$. Hence, by the bounded homotopy argument in Lemma 4.5, there exists a constant $C$ and a sequence $\left\{t_{i}\right\}$ tending to infinity so that the geodesic curves $a_{t_{i}}$ are homotopic to the closed geodesics $\alpha_{\gamma_{t_{i}}}$ by a homotopy of bounded height $C$. Taking the corresponding sequence $\left\{\gamma_{i}=\gamma_{t_{i}}\right\}$ of elements in $\Gamma_{1}$, we see that for each index $i$, the axis of $\gamma_{i}$ is within a $C$-neighborhood of the geodesic joining $x_{1}$ to $\gamma_{i}\left(x_{1}\right)$. Thus, we have, by the triangle inequality, that

$$
\left|L_{1}\left(\alpha_{\gamma_{i}}\right)-d\left(x_{1}, \gamma_{i}\left(x_{1}\right)\right)\right| \leq 2 C
$$

where $L_{1}$ is the length function on the geodesic current space $\mathcal{C}\left(\Gamma_{1}\right)$. Let $\bar{f}$ : $\mathbf{H}^{n} \rightarrow \mathbf{H}^{n}$ be a quasi-isometric (i.e. biLipschitz) extension of $f$ conjugating $\Gamma_{1}$ to $\Gamma_{2}$. By the definition of the map $f_{*}: \mathcal{C}\left(\Gamma_{1}\right) \rightarrow \mathcal{C}\left(\Gamma_{2}\right)$, if $\alpha_{\gamma_{i}}$ is induced by a closed geodesic in $\mathbf{H}^{n} / \Gamma_{1}$, then the geodesic current $f_{*}\left(\alpha_{\gamma_{i}}\right)$ is induced by a closed geodesic in $\mathbf{H}^{n} / \Gamma_{2}$ homotopic to the image under $\bar{f}$ of the closed geodesic inducing $\alpha_{\gamma_{i}}$.

We let $x_{2}=\bar{f}\left(x_{1}\right)$, then $\bar{f}\left(\gamma\left(x_{1}\right)\right)=i(\gamma)\left(\bar{f}\left(x_{1}\right)\right)=i(\gamma)\left(x_{2}\right)$. Therefore, the geodesic segment joining $x_{2}$ to $i\left(\gamma_{i}\right)\left(x_{2}\right)$ projects under the covering map to a curve that is homotopic to $f_{*}\left(\alpha_{\gamma_{i}}\right)$ (here, we regard $f_{*}\left(\alpha_{\gamma_{i}}\right)$ as the geodesic that induces the simple geodesic current), and because $\bar{f}$ is biLipschitz, there is a constant $C_{1}$ so that the resulting homotopy is a bounded homotopy of height $C_{1}$. Thus, the geodesic joining $x_{2}$ and $i\left(\gamma_{i}\right)\left(x_{2}\right)$ is in the 
$C_{1}$-neighborhood of the axis of $i\left(\gamma_{i}\right)$, and we have by another application of the triangle inequality that

$$
\left|L_{2}\left(f_{*}\left(\alpha_{\gamma_{i}}\right)\right)-d\left(x_{2}, i\left(\gamma_{i}\left(x_{2}\right)\right)\right)\right| \leq 2 C_{1} .
$$

(Here, as before, $L_{2}$ represents the length function on the space of geodesic currents $\mathcal{C}\left(\Gamma_{2}\right)$.)

Using the definition of $\tilde{R}$, and the above two inequalities, we have

$$
\tilde{R}_{12}(p) \leq \liminf _{i \rightarrow \infty} \frac{d\left(x_{2}, i\left(\gamma_{i}\right)\left(x_{2}\right)\right)}{d\left(x_{1}, \gamma_{i}\left(x_{1}\right)\right)}=\liminf _{i \rightarrow \infty} \frac{L_{2}\left(f_{*}\left(\alpha_{\gamma_{i}}\right)\right)}{L_{1}\left(\alpha_{\gamma_{i}}\right)} .
$$

By Corollary 4.9, we have that

$$
\lim _{i \rightarrow \infty} \frac{\alpha_{\gamma_{i}}}{L_{1}\left(\alpha_{\gamma_{i}}\right)}=\frac{m_{1}}{M_{1}\left(\Omega(n) / \Gamma_{1}\right)} .
$$

(Note that here $m_{1}$ is the Patterson-Sullivan geodesic current for $\Gamma_{1}$, and $M_{1}$ is the Patterson-Sullivan line element measure for $\Gamma_{1}$.) Therefore, by the continuity of $f_{*}$ (see Lemma 4.10), we have that

$$
\lim _{i \rightarrow \infty} \frac{f_{*}\left(\alpha_{\gamma_{i}}\right)}{L_{1}\left(\alpha_{\gamma_{i}}\right)}=\frac{f_{*}\left(m_{1}\right)}{M_{1}\left(\Omega(n) / \Gamma_{1}\right)} .
$$

By the continuity of the map $\alpha \rightarrow \alpha^{*}$ (see Lemma 4.2), we have that

$$
\lim _{i \rightarrow \infty} \frac{\left[f_{*}\left(\alpha_{\gamma_{i}}\right)\right]^{*}}{L_{1}\left(\alpha_{\gamma_{i}}\right)}=\frac{\left[f_{*}\left(m_{1}\right)\right]^{*}}{M_{1}\left(\Omega(n) / \Gamma_{1}\right)} .
$$

Because $\Gamma$ is convex co-compact, there exists a compact subset of $\Omega(n) / \Gamma$ that contains the support of every line element measure. Therefore, by the definition of the weak* topology, we have that

$$
\lim _{i \rightarrow \infty} \frac{\left[f_{*}\left(\alpha_{\gamma_{i}}\right)\right]^{*}\left(\Omega(n) / \Gamma_{2}\right)}{L_{1}\left(\alpha_{\gamma_{i}}\right)}=\frac{\left[f_{*}\left(m_{1}\right)\right]^{*}\left(\Omega(n) / \Gamma_{2}\right)}{M_{1}\left(\Omega(n) / \Gamma_{1}\right)} .
$$

Recall that, by definition, the length function $L_{2}$ acts on $\mathcal{C}\left(\Gamma_{2}\right)$ by $L_{2}(\alpha)=\alpha^{*}\left(\Omega(n) / \Gamma_{2}\right)$. Thus, via Proposition 4.3, we have

$$
\bar{R}_{12}\left(m_{1}\right)=\lim _{i \rightarrow \infty} \frac{L_{2}\left(f_{*}\left(\alpha_{\gamma_{i}}\right)\right)}{L_{1}\left(\alpha_{\gamma_{i}}\right)} .
$$

An immediate corollary of Theorem 4.8 is: 
Corollary 4.12. If $N_{1}$ and $N_{2}$ are isometric, then $\bar{R}_{12}\left(m_{1}\right)=\bar{R}_{21}\left(m_{2}\right)=1$.

We conjecture:

Conjecture 4.13. If $N_{i=1,2}$ are convex co-compact quasi-isometric infinite volume hyperbolic manifolds with the property that $\bar{R}_{12}\left(m_{1}\right)=\bar{R}_{21}\left(m_{2}\right)=$ 1, then the $N_{i}$ are isometric.

The Main Theorem is now an immediate consequence of Lemma 4.11 and Theorem 4.8.

Theorem 4.14 (Main Theorem). Let $\Gamma_{i} ; i=1,2$ be convex co-compact, quasi-conformally conjugate Kleinian groups. Then,

$$
\mathcal{R}_{12}=\bar{R}_{12}\left(m_{1}\right) \geq \frac{\delta\left(\Gamma_{1}\right)}{\delta\left(\Gamma_{2}\right)} .
$$

Proof. By the above lemma we have that $\tilde{R}_{12}(p) \leq \bar{R}_{12}\left(m_{1}\right)$ a.e. $\left(M_{1}\right)$. Also Lemma 3.4 shows that $\tilde{R}_{12}(p) \geq \delta\left(\Gamma_{1}\right) / \delta\left(\Gamma_{2}\right)$ a.e. $\left(M_{1}\right)$. Therefore,

$$
\mathcal{R}_{12}=\bar{R}_{12}\left(m_{1}\right) \geq \frac{\delta\left(\Gamma_{1}\right)}{\delta\left(\Gamma_{2}\right)}
$$

almost everywhere with respect to $M_{1}$, as desired. We can relate $\mathcal{R}_{12}$ and $\mathcal{R}_{21}$ through the following inequality: switching the roles of $\Gamma_{1}, \Gamma_{2}$, we obtain

$$
\frac{1}{\mathcal{R}_{12}} \leq \frac{\delta\left(\Gamma_{2}\right)}{\delta\left(\Gamma_{1}\right)} \leq \mathcal{R}_{21}
$$

\section{Acknowledgements.}

We thank the referees for their careful reading of this paper, as well as for their resulting detailed comments and corrections.

\section{References.}

[1] A. Beardon and B. Maskit, "Limit points of Kleinian groups and finitesided fundamental polyhedra," Acta Math. 132 (1974), pp. 1-12.

[2] C. Bishop and P. Jones, "Hausdorff dimension and Kleinian groups," Acta Math. 179 (1997), pp. 1-39. 
[3] Francis Bonahon "The geometry of Teichmüller space via geodesic currents," Invent. Math. 92 (1988), pp. 139-162.

[4] B.H. Bowditch, "Geometrical finiteness for hyperbolic groups," J. Funct. Anal. 113 (1993), pp. 245-317.

[5] M. Bridgeman and E.C. Taylor, "Length distortion and the Hausdorff dimension of Kleinian groups," Amer. J. Math 122 (3) (2000), pp. 465482 .

[6] M. Bridgeman and E.C. Taylor, "The analytic properties of length distortion: an extension of the Weil-Petersson metric to quasi-Fuchsian space," preprint.

[7] R. Canary, D. Epstein and P. Green, "Notes on notes of Thurston," in Analytical and Geometrical Aspects of Hyperbolic Space, Cambridge University Press, 1987.

[8] A. Douady and C. Earle, "Conformally natural extensions of homeomorphisms of the circle," Acta Math. 157 (1986), pp. 23-48.

[9] B. Maskit, Kleinian Groups, Springer-Verlag, 1987.

[10] P. Nicholls, The Ergodic Theory of Discrete Groups, Cambridge University Press, 1989.

[11] S. Patterson, "The limit set of a Fuchsian group," Acta Math. 136 (1976), pp. 241-273.

[12] H. Reimann, "Invariant extension of quasi-conformal deformations," Ann. Acad. Sci. Fenn. Ser. AI Math., 20 (1985), pp. 477-492.

[13] D. Sullivan, "The density at infinity of a discrete group of hyperbolic motions," Publ. Math. IHES 50 (1979), pp. 171-202.

[14] D. Sullivan, "Entropy, Hausdorff measures old and new, and limit sets of geometrically finite Kleinian groups," Acta Math. 153 (1984), pp. 259-277.

[15] W.P. Thurston, The Geometry and Topology of 3-Manifolds, Lecture Notes, Princeton University, 1979.

[16] P. Tukia, "The Hausdorff dimension of the limit set of a geometrically finite Kleinian group," Acta Math. 152 (1984), pp. 127-140. 
[17] S. Wolpert, "Thurston's Riemannian metric for Teichmüller space," J. Diff. Geom. 23 (1986), pp. 173-174.

Department of Mathematics

Boston College

Chestnut Hill, MA 02467

E-mail address: bridgem@bc.edu

Department of Mathematics

WESLEYAN UNIVERSITY MidDLETOWN

CT 06459-0128

E-mail address: ectaylor@wesleyan.edu

ReCEIVED July 11, 2000. 